\title{
Measurement of displacement cross section of structural materials utilized in the proton accelerator facilities with the kinematic energy above $400 \mathrm{MeV}$
}

\author{
Shin-ichiro MEIGO ${ }^{1, *}$, Hiroki MATSUDA ${ }^{1,}$, Yosuke IWAMOTO $^{1,3}$, Makoto $^{2}$ YOSHIDA $^{2,}$, Shoichi HASEGAWA ${ }^{1,}$, \\ Fujio MAEKAWA ${ }^{1,}$, Hiroki IWAMOTO ${ }^{1}$, Tatsushi NAKAMOTO $^{2}$, Taku ISHIDA $^{2,}$, and Shunsuke MAKIMURA ${ }^{2,}$ \\ ${ }^{1}$ J-PARC Center, Japan Atomic Energy Agency, 2-4 Shirakata, Tokai, Naka, Ibaraki 319-1195, Japan \\ ${ }^{2}$ High Energy Accelerator Research Organization, 1-1 Oho, Tsukuba, Ibaraki 305-0801, Japan \\ ${ }^{3}$ Nuclear Science and Engineering Center, Japan Atomic Energy Agency, 2-4 Shirakata, Tokai, Naka, Ibaraki 319-1195, Japan
}

\begin{abstract}
For damage estimation of structural material in the accelerator facility, displacement per atom (DPA) is widely employed as an index of the damage calculated based on the displacement cross section obtained with the calculation model. Although the DPA is employed as the standard, the experimental data of displacement cross section are scarce for a proton in the energy region above $20 \mathrm{MeV}$. Among the calculation models, the difference exists about 8 times so that experimental data of the displacement cross section is crucial to validate the model. To obtain the displacement cross section, we conducted the experiment in J-PARC. As a preliminary result, the displacement cross section of copper was successfully obtained for $3-\mathrm{GeV}$ proton. The present results showed that the widely utilized the Norgertt-Robinson-Torrens (NRT) model overestimates the cross section as suggested by the previous experiment for protons with lower energy.
\end{abstract}

\section{Introduction}

To decrease hazard of the radioactive waste produced in a nuclear reactor, Japan Atomic Energy Agency (JAEA) proposes the Accelerator Driven System (ADS) with extremely high power accelerator such as $30 \mathrm{MW}$ with proton kinetic energy of $1.5 \mathrm{GeV}$. A lead-bismuth eutectic (LBE) is one of a candidate of the target, which simultaneously plays the role of the coolant. In the design of the ADS, damage to the beam intercepting material is one of a critical issue. In other high-intensity accelerator facilities, beam intercepting material also plays essential roles. At Material Life science experimental Facility (MLF) [1, 2] in J-PARC[3], an aluminum alloy is utilized as beam window[4] separating between high vacuum area and target station. The T2K[5] collaboration in J-PARC uses the titanium alloy as the beam window. To operate high power accelerator with confidence, damage estimation of the target material is essential. For the quantitative specification of the damage to the target material, displacement per atom (DPA) is employed in general. The DPA is widely used for the estimation of damage nuclear reactor and fusion reactor as well.

The DPA is estimated by the particle flux multiplied displacement cross section. The cross section is usually obtained by Norgertt-Robinson-Torrens (NRT) model[6]. In the lower energy region than $20 \mathrm{MeV}$, the displacement cross section for proton can predict well because the displacement is mainly caused by Coulomb force. For the neutrons in lower energy, the DPA can be estimated

*e-mail: meigo.shinichiro@jaea.go.jp with reasonable accuracy as well, because the displacement cross section can be simply denoted by the elastic and inelastic scattering. For those scattering, the cross section and outgoing energy of the second particle are well known. The calculation method of the displacement cross section has been established for the low-energy regions where nuclear reactions do not produce secondary particles. However, for the protons in the high energy region above $20 \mathrm{MeV}$, the experimental data of displacement cross section are scarce. Therefore, the displacement cross section has not been studied well. Since many reaction channels open above $20 \mathrm{MeV}$, the calculation codes based on the intra-nuclear cascade model are utilized to obtain the cross section. So far, the experimental data carried out Kyoto University[7] and BNL/AGS[8] are available above $20 \mathrm{MeV}$. It was reported that the displacement cross section of tungsten calculated with the MARS code[9] has 8 times difference in various calculation models. If the damage of the intercepting material is dominated displacement, 8 times of ambiguity for the displacement cross section makes 8 times the uncertainty of lifetime estimation. For validation and improvement of the estimation of the DPA, the experimental data are crucial. Since the experimental data of the DPA are scarce, the experimental data are required in the proton energy region above $20 \mathrm{MeV}$ to improve the displacement cross section.

\section{Experiment}

For the displacement cross section measurement, we conducted the experiment in the beam transport line called 
3NBT in J-PARC. A vacuum chamber with cryocooler installed at the front of the beam dump of 3-GeV Rapid Cycling Synchrotron (RCS). Since no space was left in the beamline introduced to the beam dump, the present experiment equipment was installed in the beamline, where the high-intensity proton beam may deliver to the spallation neutron source in MLF. Although the proton beam with low intensity was irradiated to sustain a cryogenic temperature of the sample, the additional interlock of the accelerator was required for the experiment due to the safety reason. At the RCS, the extracted proton kinematic energy can vary from $0.4 \mathrm{GeV}$ to $3 \mathrm{GeV}$ with altering the timing of the extraction kicker magnet. With the change of the rigidity of the following magnets, the beam was introduced to the sample.

\subsection{Procedure of displacement cross section measurement}

For observation of the damage in the sample, the irradiated sample is required to be cooled cryogenic temperature (around $10 \mathrm{~K}$ ), where recombination of Frenkel pairs by thermal motion is well suppressed. With the observation of the resistivity change $\Delta \rho_{C u}$ due to irradiation under cryogenic temperature, the experimental displacement cross section $\sigma_{\text {exp }}(E)$ is given by the following,

$$
\sigma_{e x p}(E)=\Delta \rho_{C u} /\left(\overline{\phi(E)} \rho_{F P}\right),
$$

where $\overline{\phi(E)}$ is average proton flux over the sample surface accumulated for irradiation time, $\rho_{F P}$ is the resistivity change per Frenkel-pair density for a particular metal. In this work, $\rho_{F P}$ was defined to $2.2 \times 10^{-6} \Omega \mathrm{m}$ from other study[10].

The increase in electrical resistivity due to highenergy protons provides straightforward information such as degradation of the stabilizer of the superconductor. The degradation compromises quench protection and increase magnetothermal instability, for the superconducting accelerator magnet utilized in the hadron collider such as HighLuminosity Large Hadron Collider (LHC) in CERN.

\subsection{Measurement of displacement cross section at 3-GeV beam dump in J-PARC}

A vacuum chamber, as shown in Fig. 1 was installed in front of the $3-\mathrm{GeV}$ beam dump. The schematic of the chamber and sample is shown in Fig. 2. The chamber was deployed Gifford-McMahon (GM) cryocooler (Sumitomo Heavy Industries RDK-408D2) which cooled the sample through a copper rod and sample holder made of aluminum. The assembly of cryocooler and sample was placed on a movable stage to control irradiation. In order to compare the previous experiments, copper was selected as the sample. Before installation, the copper sample was annelid about $800^{\circ} \mathrm{C}$ to eliminate the defect of the lattice. The resistance of the sample was measured by the voltmeter (Keithley 6221) and current source (Keithley 2185). The sample wire at the terminal (i.e., potential point) was connected to both current source and voltmeter for compensation of cable resistance between the sample and the instruments. A current of $\pm 100 \mathrm{~mA}$ was supplied to the copper wire from the current source with a duration of 1 $\mathrm{s}$ for every $30 \mathrm{~s}$. In order to improve accuracy, the delta mode with the polarity exchange was applied with a frequency of $10 \mathrm{~Hz}$. The voltage of the copper wire was read once every minute. The precision of this resistance measurement was $\pm 0.01 \mu \Omega$, corresponding to a resistivity of $\pm 3 \mathrm{f} \Omega \mathrm{m}$.

Table 1 summarizes the electrical resistivity measurements of the sample. The residual resistivity ratio (RRR) of the sample was measured between a room and cryogenic temperature. The CX1050-SD Cernox resistance thermometer on the sample holder was calibrated in the temperature region 4-100 $\mathrm{K}$ and the silicon thermometer on the copper column was calibrated between $4 \mathrm{~K}$ and room temperature. The electrical resistivity of the sample $\rho_{C u}$ is expressed as follows:

$$
\rho_{C u}=R A / L,
$$

where $R[\Omega]$ is the measured electrical resistance, $\mathrm{A}$ is the area of the sample $\left(4.9 \times 10^{-8}\left[\mathrm{~m}^{2}\right]\right)$, and $L[\mathrm{~m}]$ is the length between two potential points $(40 \mathrm{~mm})$. The minimum temperature on the sample holder was $20 \mathrm{~K}$, although the temperature of near the cold head was $4.6 \mathrm{~K}$. The main reason for the different temperatures was insufficient thermal contact between the copper rod and the holder.

The beam intensity was observed by the wellcalibrated current transformer. The beam width was observed with the multi-wire-profile-monitor (MWPM)[11, 12] placed near the sample, which was 4.0 and $5.0 \mathrm{~mm}$ for the horizontal and vertical direction, respectively, in $1 \sigma$. From the result of the beam measurement, the average flux was derived as $1.98 \times 18 \mathrm{~m}^{-2}$.

Table 1. Characteristics of copper sample.

\begin{tabular}{ll}
\hline Shape & Single wire \\
Diameter $[\mu \mathrm{m}]$ & 250 \\
Length between potential points $[\mathrm{mm}]$ & 40 \\
Purity [\%] & 99.999 \\
Resistivity at $298 \mathrm{~K}[\Omega \mathrm{m}]$ & $1.67 \times 10^{-8}$ \\
Resistivity at $20 \mathrm{~K}[\Omega \mathrm{m}]$ & $7.36 \times 10^{-11}$ \\
Residual Resistivity Ratio (RRR) & 227 \\
\hline
\end{tabular}

\section{Result and discussion}

\subsection{Result of the present experiment}

The damage rate of copper irradiated with a proton of the present result is shown in Table 2 compared with the previous experiment carried out for lower energy proton. Using the present damage rate and Eq. (1), the displacement cross section of the proton was obtained, which was $970 \mathrm{~b}$ of $\mathrm{Cu}$ at $3 \mathrm{GeV}$. 


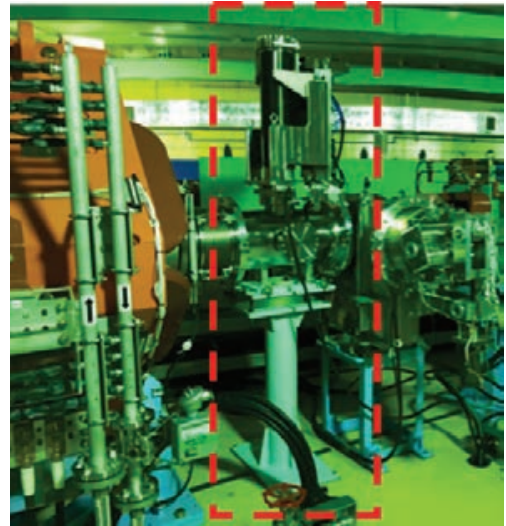

Figure 1. Vacuum chamber with GM cryocooler and sample mounted on movable stage or the measurement displacement cross section placed at the beamline shown in hatched line.

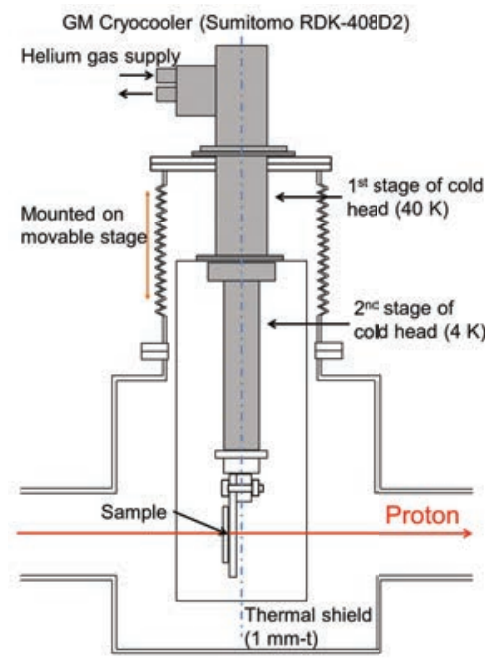

Figure 2. Schematic drawing of equipments utilized in the present experiment.

Table 2. Experimental resistance change rate to one proton induced.

\begin{tabular}{lcc}
\hline Experiment & $\begin{array}{c}\text { Energy } \\
{[\mathrm{GeV}]}\end{array}$ & $\begin{array}{c}\text { Damage rate } \\
{\left[\times 10^{-31} \Omega \mathrm{m}^{3} / \text { proton }\right]}\end{array}$ \\
KURRI[7] & 0.13 & 3.41 \\
AGS[8] & 1.94 & 3.66 \\
Present(J-PARC) & 3.0 & 2.13 \\
\hline
\end{tabular}

\subsection{Calculation of displacement cross section}

The displacement cross section, which is defined as following equation obtained with the calculation based on intra-nuclear cascade model,

$$
\sigma_{\text {disp-calc }}(E)=\sum_{i} \int_{E_{d}}^{T_{i}^{\max }} v\left(T_{i}\right) d \sigma / d T_{i},
$$

where $\mathrm{E}$ is the kinetic energy of projectile, $d \sigma / d T_{i}$ is recoil atom kinetic energy distribution, $T_{i}$ is the kinetic energy of recoil particle $i$ valid to maximum of $T_{i}^{\max }, E_{d}$ is the effective threshold displacement energy, and $v\left(T_{i}\right)$ is the num- ber of defects (Frenkel pairs) showing the defect production efficiency defining by a vacancy and a self-interstitial atom in the irradiated material, which is widely utilized by Norgertt-Robinson-Torrens (NRT) model[6]. Calculation result was obtained with PHITS code[13]. The displacement cross section, which is defined as following equation obtained with the calculation based on intra-nuclear cascade model,

$$
\sigma_{\text {disp-calc }}(E)=\sum_{i} \int_{E_{d}}^{T_{i}^{\max }} v\left(T_{i}\right) d \sigma / d T_{i},
$$

For the calculation of the displacement, two components of the process were applied in PHITS. One is a Coulomb's scattering cross-section between the charged particle of projectile and target atom. The NRT-dpa crosssection is obtained by finding the integral between the Coulomb's scattering cross-sections and number of atomic displacements. The other component is a nuclear reaction including elastic and inelastic scattering with a nucleus. The number of displacement atoms with the NRT model is expressed as follows:

$$
N_{N R T}=0.8 E_{d} / 2 E_{D}
$$

where $E_{d}$ is the damage energy transferred to lattice atoms and $E_{D}$ is the threshold displacement energy. The NRT model cross section for the projectile and target atom are obtained using the following equation:

$$
\sigma_{N R T}=\int_{t_{d}}^{t_{\max }} \frac{d \sigma_{s c}}{d t} N_{N R T} d t,
$$

where $t$ is the dimensionless collision parameter, which includes information regarding the kinetic energy of recoil. The maximum kinetic energy of recoil, $t_{\max }$, is determined by the kinematics between the incident charged particle and target atom. The parameter of $t_{d}$ is the dimensionless energy of the threshold displacement energy $E_{D}$, and $d \sigma_{s c} / d t$ is the Coulomb's scattering cross-section.

In the energy range of an incident particle above 10 $\mathrm{MeV} / \mathrm{u}$, secondaries are produced by nuclear reactions. The cross section for secondary particles was obtained using the Monte Carlo calculation of charged-particle incident reactions on a thin sample, which is expressed as follows:

$$
\sigma_{N R T}=D P A / \phi=\sum_{i=1}^{N} N_{N R T i} / n_{0} A \phi,
$$

where $n_{0}$ is the Avogadro number of a target and $A$ is the atomic number of a target. $\phi$ is the beam fluence on a target, $i$ denotes a number indicating secondaries produced by a nuclear reaction. $N_{N R T}$ is calculated using Eq. (5) on an event-by-event basis. The damage energy transferred to lattice atoms in Eq. (5) is obtained using the kinetic energy of secondaries produced by the direct and static models. The geometry of the target was a cylinder with diameter and thickness of $10 \mathrm{~mm}$ and $1 \mu \mathrm{m}$, respectively, and that of a beam was a circle with a $10-\mathrm{mm}$ diameter. In the calculations, the kinetic energies of secondaries are scored when the secondaries are produced in a sample. 
Recently, Nordlund et al. developed the athermal recombination-corrected (arc) displacement per atom (dpa) function obtained with MD simulations, which provides more physically realistic descriptions of primary defect creation[14]. The arc-dpa cross-section was calculated using the displacement efficiency function of $\zeta$, whic is obtained from the molecular dynamics (MD) simulation study[14].

$$
\begin{gathered}
N_{\text {arc }}=0.8 E_{d} \zeta\left(E_{d}\right) / 2 E_{D} \\
\zeta\left(E_{d}\right)=\frac{1-c}{\left(2 E_{D} / 0.8\right)^{b}} E_{d}^{b}+c
\end{gathered}
$$

In the parameters of $\mathrm{b}$ and $\mathrm{c}$ are the tabulated parameters for each nucleus obtained by the molecular study given by Nordlund et al [14]. The $E_{D}$ value was determined as 27 $\mathrm{eV}$. The cross-section of arc-dpa was also calculated with PHITS

\subsection{Comparison between experiment and calculation}

In Fig. 3, the present preliminary result of the displacement cross section is compared with the previous experimental data and the calculation. The present data showed overestimation about 4 times of the calculation with the NRT model as similar to the previous experiment carried out lower energy [7, 8]. It was also shown that the arc-dpa with Nordlund[14] model showed remarkably good agreement with the experimental data. By the accumulation of the experimental data in future work, the accuracy of the calculation can be expected to be improved.

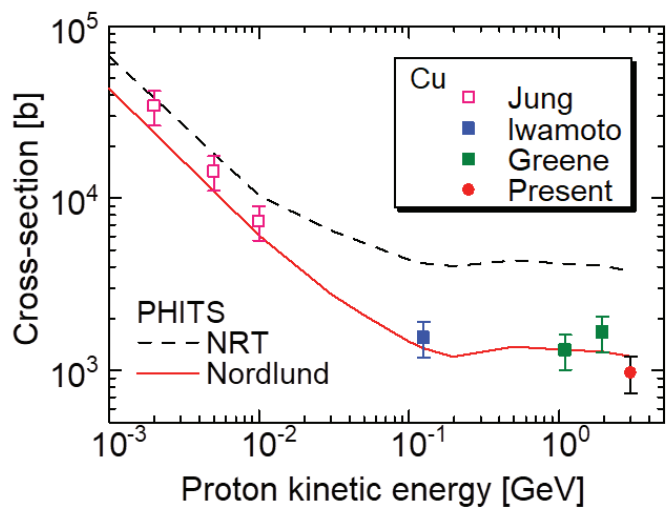

Figure 3. Comparison of the present experiment data with the previous experiment $[7,8,15]$ and calculation results with PHITS as a function of kinetic energy.

\section{Conclusion}

For estimation of target materials used at high-intensity proton accelerator, displacement cross section experiment was carried out in J-PARC. As a preliminary result, the displacement cross section of copper for $3-\mathrm{GeV}$ proton was successfully obtained, which was obtained by the change of resistivity for the irradiated sample with cryotemperature. The present results showed an overestimation of the cross section with widely employed the Norgertt-Robinson-Torrens (NRT) model, which was suggested by the previous experiment for the proton projectile with lower energy than the present. A similar chamber will be installed in front of beam dump for the $30-\mathrm{GeV}$ synchrotron to obtain the cross section for the proton energy range between $3 \mathrm{GeV}$ and $30 \mathrm{GeV}$. After compiling the data, the cross section from 0.4 to $30 \mathrm{GeV}$, which will help improve the calculation model for displacement cross section.

\section{Acknowledgment}

The present study includes the results of Measurement of displacement cross section at J-PARC for structural material utilized at ADS entrusted to JAEA by the Ministry of Education, Culture, Sports, Science and Technology of Japan (MEXT).

\section{References}

[1] Y. Ikeda, Nucl. Instrum. Meth. A600, 1-4 (2009).

[2] Y. Miyake, et al., Physica B 484, 374-375 (2006).

[3] The Joint Project Team of JAERI and KEK, JAERITech 99-56, 1999.

[4] S. Meigo, et al., J. Nucl. Mater. 450, 141-146 (2014).

[5] K. Abe, et al., Nucl. Instrum. Meth. A659, 106-136 (2011).

[6] M. J. Norgett, M. T. Robinson, I. M. Torrens, Nucl. Eng. Des. 33 , 50-54 (1975).

[7] Y. Iwamoto, et al., J. Nucl. Mater. 458, 369-375 (2015)

[8] G. A. Greene, et al., Proc. of 6th Int. Meet. on Nucl. Application of Accel. Tech. (AccApp03), Ja Grange Park, Illinois, USA, pp. 881-892, (2004).

[9] N. Mokhov, et al., "MARS15 Developments Related to Beam-Induced Effects in Targets", 6th HighPower Targetry Workshop, Oxford, UK, April 11-15 (2016).

[10] H. G. Haubold, D. Martinsen, , J. Nucl. Mater. 69 and 70, 644-649 (1978).

[11] S. Meigo, et al., Nucl. Instrum. Meth. A562, 569-572 (2006).

[12] S. Meigo, et al., Nucl. Instrum. Meth. A600, 41-43 (2009).

[13] Y. Iwamoto, H. Iwamoto, M. Harada, K. Niita, J. Nucl. Sci. Technol. 51, 98-107 (2014).

[14] K. Nordlund, et al., Nature Communications 9, 1084 (2018)

[15] P. Jung, J. Nucl. Mater. 117, $70-77$ (1983). 